\title{
SELF-ORGANIZATION AND AUTONOMOUS NETWORK SURVEY
}

\author{
Houneida SAKLY \\ COSMOS Laboratory -National Institute of Computer Sciences - \\ Campus University of Mannouba -Tunisia
}

\begin{abstract}
Purpose

The autonomic network gathers several aspects of Self-organization, which is depicted, into different autonomous function such as the Self-configuration, the Self-optimization, the Self-repair, the Selfprotection, and the Self-cure. The latter is considered as one of the autonomous functions wished of a system network, which could be described by autonomous behavior is realized by structures of the control loops and loop of control.
\end{abstract}

\section{Issue}

These various aspects require an architectural framework, which integrates several protocols of telecommunication as well as programs proactive and reagents for the prevention and the resolution of the unforeseen problems. To ensure a reduction of the loops of the operator, we need good management per policy. An autonomic vision must guarantee the safety and the availability of the resources for the endusers by respecting the constraints of QoS and the deadlines for replies.

\section{Goals}

Depict the challenges, the interests, and the anomalies and compare the autonomic approaches of the networks which consist of discovering neighbor's in the self-organizing wireless network. These points are considered the cornerstone of extending autonomous coverage and improving performance. However, the objective is to detect the hidden node in the neighbor discovery procedure based on the simple flood caused by the uncoordinated transmission, which can cause an unnecessary loss of energy.

\section{KEYWORDS}

Autonomic Network, Self-configuration, Self-optimization, Self-protection, Self-cure, Self-configuration, QoS, response time, safety

\section{INTRODUCTION}

These Technologies of modern networks such as BAM, WAN, and LAN, are considered complicated from a management point of view. It will be almost impossible for human administrators to supervise them. To control this complication, IBM introduces an approach promising to aim to create systems of Self-management. This approach of the networks autonomic aims to conceive which data-processing equipment in the measurement of Selfadaptation to meet high level aims definitely by the human operator. To obtain autonomous behaviors, the architecture of these networks is conceived to implement several forms of policies,

DOI: $10.5121 /$ ijnsa.2020.12102 
International Journal of Network Security \& Its Applications (IJNSA) Vol. 12, No.1, January 2020

which have like objective principal; form tools allowing a greater reactivity the level of the networks and services, and to guarantee their availability with the end-users. According to welldefined directives of management, the policies must describe autonomous behaviors to make the follow-up of the equipment of network, which sensitized to achieve a given goal to highlight the advantages of architecture and the continuity of the policies aiming at introducing autonomous management into a multiservice network IP [1] [2]. The rest of this paper is organized as follows: the description of the issue, the architecture and the concept of anatomical networks as well as the main objectives and their features.

\section{RELATED WORK AND APPROACHES FOR THE AUTONOMOUS NETWORKS}

Table 1: Existing approaches for the autonomous networks

\begin{tabular}{|c|c|c|c|c|}
\hline & $\begin{array}{l}\text { DMA } \\
\text { approaches: } \\
\text { autonomous } \\
\text { management } \\
\text { distributed }^{1}\end{array}$ & $\begin{array}{l}\text { Approaches estimate } \\
\text { of confidence via the } \\
\text { passage of messages }\end{array}$ & $\begin{array}{l}\text { Approaches } \\
\text { diffusion of the } \\
\text { context automated } \\
\text { by the means of a } \\
\text { filter of } \\
\text { subscription }{ }^{3}\end{array}$ & $\begin{array}{l}\text { Approaches evolutionary } \\
\text { and embryogenesis }{ }^{4}\end{array}$ \\
\hline$[3]^{1}$ & $\begin{array}{l}\text { composed of a } \\
\text { whole of the } \\
\text { agent which } \\
\text { resides in a } \\
\text { basic field }\end{array}$ & $\begin{array}{l}\text { based on the theory } \\
\text { of the estimate like a } \\
\text { problem of inference } \\
\text { with the Markovian } \\
\text { chain }\end{array}$ & $\begin{array}{l}\text { management is } \\
\text { supposed to be } \\
\text { automated by } \\
\text { introducing the } \\
\text { autonomic layer of } \\
\text { the distributed } \\
\text { element of } \\
\text { management }\end{array}$ & $\begin{array}{l}\text { Monitoring and control of } \\
\text { the performance of the } \\
\text { component network }\end{array}$ \\
\hline$[4]^{2}$ & $\begin{array}{l}\text { The agents } \\
\text { move towards } \\
\text { another field } \\
\text { under the } \\
\text { request of the } \\
\text { nodes to exert a } \\
\text { functionality }\end{array}$ & $\begin{array}{l}\text { Application of a } \\
\text { distributed algorithm } \\
\text { based on the } \\
\text { exchange of the } \\
\text { technical messages } \\
\text { of passages }\end{array}$ & $\begin{array}{l}\text { the requirements of } \\
\text { the context of } \\
\text { decision making are } \\
\text { converted and } \\
\text { stored }\end{array}$ & Analyzes performances \\
\hline$[5]^{3}$ & $\begin{array}{l}\text { the agents can } \\
\text { destroy their } \\
\text { spots once is } \\
\text { finished }\end{array}$ & $\begin{array}{l}\text { Combination of the } \\
\text { theory of MARKOV } \\
\text { RANDOM FIELDS } \\
\text { with the algorithm of } \\
\text { the technical } \\
\text { messages of } \\
\text { passages to make the } \\
\text { development of an } \\
\text { algorithm of } \\
\text { distribution of } \\
\text { information and } \\
\text { fusion }\end{array}$ & $\begin{array}{l}\text { generation of an } \\
\text { algorithm for the } \\
\text { filters of } \\
\text { subscriptions }\end{array}$ & $\begin{array}{l}\text { Planning and operation of } \\
\text { the changes in the software } \\
\text { components and the } \\
\text { structure }\end{array}$ \\
\hline
\end{tabular}


International Journal of Network Security \& Its Applications (IJNSA) Vol. 12, No.1, January 2020

\begin{tabular}{lllll}
\hline [6] $^{4}$ & $\begin{array}{l}\text { the agents can } \\
\text { migrate towards } \\
\text { the nodes in a } \\
\text { temporary way }\end{array}$ & $\begin{array}{l}\text { The messages are } \\
\text { not regarded as } \\
\text { abstract intermediate } \\
\text { results but real } \\
\text { messages of } \\
\text { exchange via the } \\
\text { network nodes. }\end{array}$ & $\begin{array}{l}\text { knowledge of the } \\
\text { models is } \\
\text { summarized to } \\
\text { maintain the size of } \\
\text { the controlled } \\
\text { model }\end{array}$ & $\begin{array}{l}\text { Execution of the new } \\
\text { planned solution }\end{array}$ \\
& & \\
\hline
\end{tabular}

\section{ISSUE}

The location of the current networks such as ROUND OF APPLAUSE (Body Area NR), SIDE (Personal Area NR), VAN (Vehicular Area NR), HAN (Home NR), LAN EDGE (Committee Area NR), AMAM, VAN, became increasingly complicated because of the false constraints of QOS (Quality Of Services) .la complexity of technologies due to the heterogeneity of the components the main issue of the networks it is the complexity of management which requires from $20 \%$ to $50 \%$ of time for the determination and the resolution of the problems. Whereas $40 \%$ of the breakdowns of services are due to problems of configuration.

Table 2: Comparison enters the needs, the challenges and the evolution [1, 2,3,4,5]

\begin{tabular}{lll}
\hline \multicolumn{1}{c}{ Needs } & \multicolumn{1}{c}{ Challenges } & \multicolumn{1}{c}{ Evolution } \\
\hline $\begin{array}{l}\text { To reduce the cost of } \\
\text { maintenance }\end{array}$ & $\begin{array}{l}\text { autonomic } \\
\text { distribution of } \\
\text { management }\end{array}$ & $\begin{array}{l}\text { A reduction in the intervention of human } \\
\text { resources in the operation and the network } \\
\text { management }\end{array}$ \\
$\begin{array}{l}\text { To provide and guarantee the } \\
\text { availability of the networks } \\
\text { and the services }\end{array}$ & $\begin{array}{l}\text { knowledge of } \\
\text { the data }\end{array}$ & $\begin{array}{l}\text { The elements of the network must be } \\
\text { integrated into their environment and interact } \\
\text { with the elements in place }\end{array}$ \\
$\begin{array}{l}\text { Contract of self-management } \\
\text { To provide tools allowing a } \\
\text { greater reactivity level of the } \\
\text { networks and the services. }\end{array}$ & $\begin{array}{l}\text { Self-adaptation } \\
\text { speed, QoS, }\end{array}$ & $\begin{array}{l}\text { QOG systems manage themselves, in a } \\
\text { distributed way, following objectives establish } \\
\text { by the administrator }\end{array}$ \\
\hline $\begin{array}{l}\text { They must be able to adapt to their new } \\
\text { environment and to continue to Self-Attempt } \\
\text { out the goals which were assigned to them }\end{array}$ \\
\hline
\end{tabular}

\section{NETWORK ARCHITECTURE}

The concept of autonomic computing is a concept suggested by IBM in 2001 and perceived as the model of reference. Autonomic networking and the autonomic communication are two terms, which are in the same mobility and influenced by the concept of autonomic computing.

The anatomic network can make their Self-management and the Self-communication without the intervention of the human administrators. While adapting to the dynamic evolution of the state of the resources managed, the networks autonomic maintain and adjust their behaviors according to 
International Journal of Network Security \& Its Applications (IJNSA) Vol. 12, No.1, January 2020

the internal, external changes of the requests, the objectives, and the software or material breakdowns malicious.

\section{CONCEPT OF ANATOMic NeTWORK}

ANEMA (Autonomic Network Management Structures) defines autonomous network management which implements a political whole of concept. ANEMA is organizing into two layers:

- Definition of the objectives

- Realization of the objectives

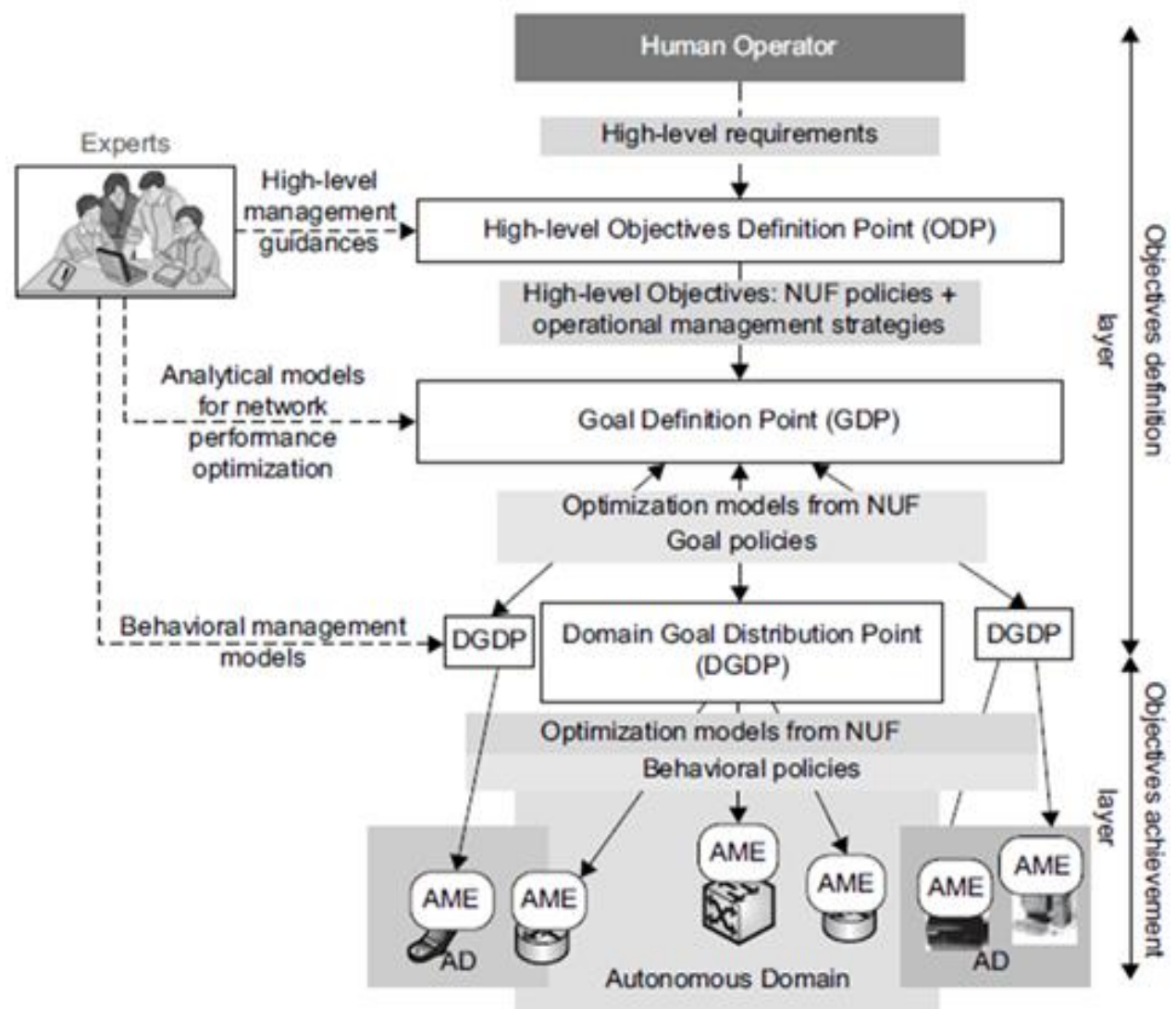

AME $=$ Autonomic Management Element, NUF $=$ Network Utility Function

Figure 1: Whole sight of ANEMA [2]

\subsection{Aims Description}

The principal component of the layer it is the ODP (Objective of Point of Definition). It makes it possible for the administrators to introduce their high requirements, and to the experts to introduce high-level management. In the ODP, the high-level requirements are transformed into 
International Journal of Network Security \& Its Applications (IJNSA) Vol. 12, No.1, January 2020

NUF (Function Utility of the network, while the guiding lines of management are transformed into abstract strategies of management. NUFs present the rules or policies that describe the performances of the network from the human point of view. They are used to express the functionalities of the network in terms of a function of optimization, while the strategies of management describe the high-level guidelines which are necessary to the chart. For specific management of architecture, the NUF implements within the infrastructure of the target network. The ODP transmits the formulas and the strategies of the NUF towards the GDP (Objective of Point of Definition) which analyzes them and identifies the policies of the related goals. To facilitate the distribution of the policies of the objectives, the infrastructure of the network is divided into several fields. Each field is supervised by coordinators called DGDP (Domain Gout Distribution Definition Not). GPP transmitted specificities of the objectives and the total modules of total obtaining NUF towards the DGPP [7].

\subsection{Goals Realization}

This layer contains a whole of the intervals called GAPs. Each GAP constitutes an entity that behaves autonomously while trying to reach the high-level requirements and by taking account of the policies and the models of optimization. Indeed, these (GAPs) can make its own decisions to Self-try out elementary monitoring, the analysis of the data elements, the planning and the execution of the capacities. Moreover, the intervals (GAPs) have also able to question with its environment and to communicate with other gaps [7].

\subsection{Simulation-Based Scenario}

The scenario is shown in Figure2. makes it possible to add combination gateways for each point of the network to increase resilience and capacity and optimize routing and overall latency.

Human intervention for the configuration and planning of the network is not compulsory since this concept is reserved for the network classified as "backhaul".

The use of standardized client protocols automates access by client devices. User-driven experience and engagement (subject to a different discussion) highlight the distinct component: in this case, the autonomous network is triggered autonomously if the backhaul is connected and the users connect to the network.

The novelty is to make an intelligent decision, which requires an in-depth analysis concerning the addition of backhaul links and the modification of the quality of service for users. 


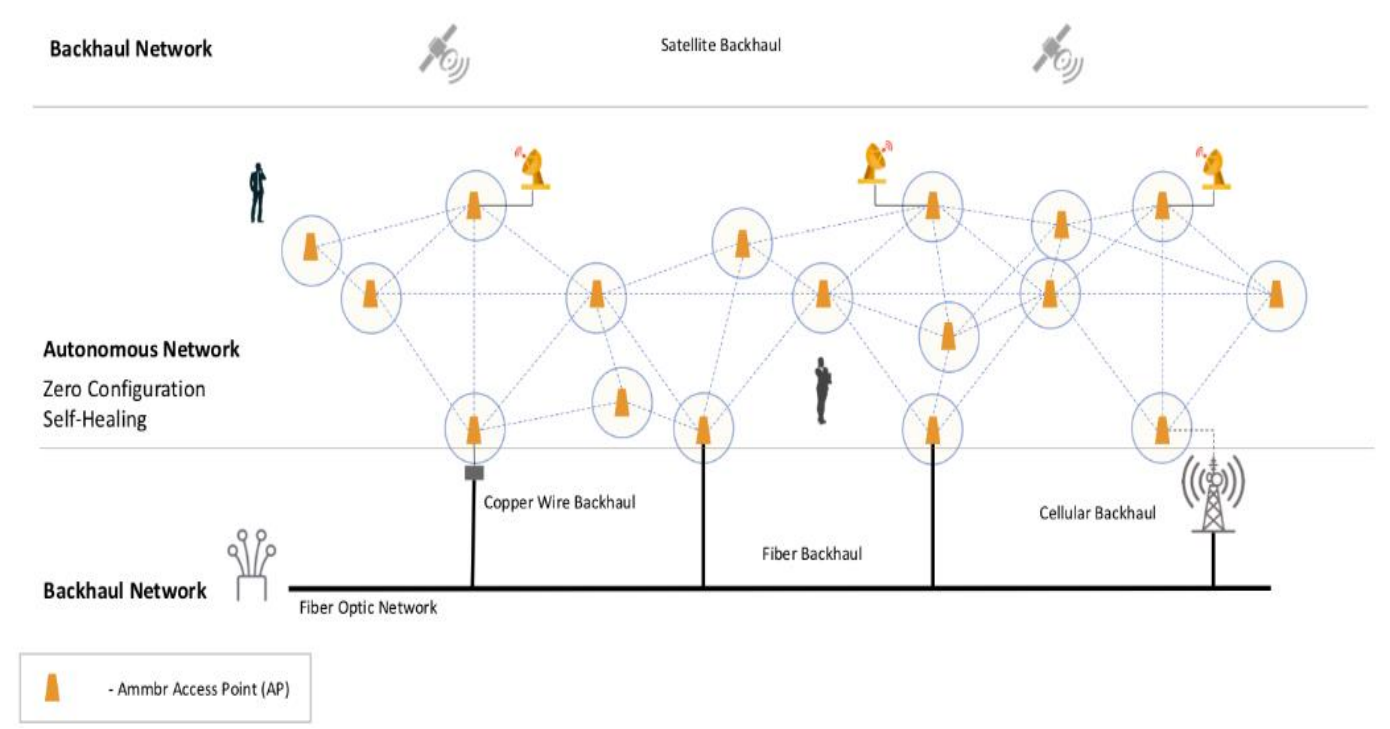

Figure.2: Model simulation

\section{Main Features of Autonomic Networks}

The gasoline of the networks autonomic (component system + component network) is the Selfcommunication which enables him to make the adoption of the services and the resources and what they offer according to the evolution of the user's needs, the conditions of the environment and the objectives of a business.

\subsection{Self-Repair}

This function is used for detecting, diagnosing and repairing automatically the anomalies of the system. The detection of the anomalies plays a vital part in the autonomous networks. Self-repair makes it possible to make the diagnosis of the accidental errors of the system. The reasons for Self management of the autonomous networks and the detection of the anomalies are narrowly integrated with the answer and the realization of the automatic functionality of defense. Moreover, the anomalies of the system networks were also modified for the autonomous network by the exploration of the particular characteristics of the network. 
International Journal of Network Security \& Its Applications (IJNSA) Vol. 12, No.1, January 2020

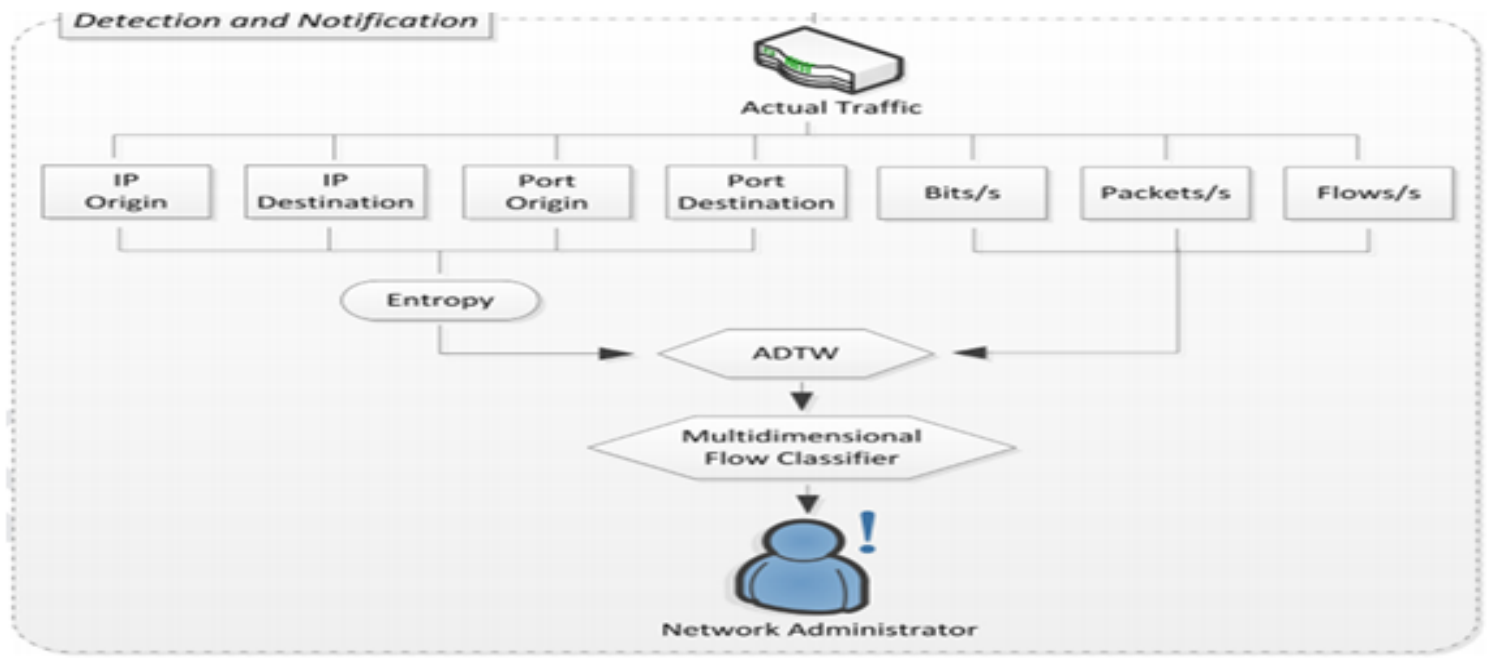

Figure 3: Detection of the anomalies of the system of component [8]

The detector of an anomaly in the networks autonomic makes its Self-management. Its operations are transparent for the subjacent networks. In particular, an $\mathrm{AD}$ necessarily does not manage with protocols of routing; it supervises and analyzes the errors of interest.

Since each $\mathrm{AD}$ is conscious only of its local environment. Its cover of detection is limited. Formally, the behavior of an $\mathrm{AD}$ characterizes its interaction with the context of exploitation according to:

- $\quad$ AD supervises the behavior of nodes (package of network and network of routing

- $\mathrm{AD}$ functions the model of detection and estimates the state of the network via the collected observations if a consensus is reached.

- VAD takes appropriate measures for the anomalies

$\mathrm{AD}$ adjusts its behavior for the next turn of detection at the time of reception of an external signal under the whole of the state of the network.[1,7]

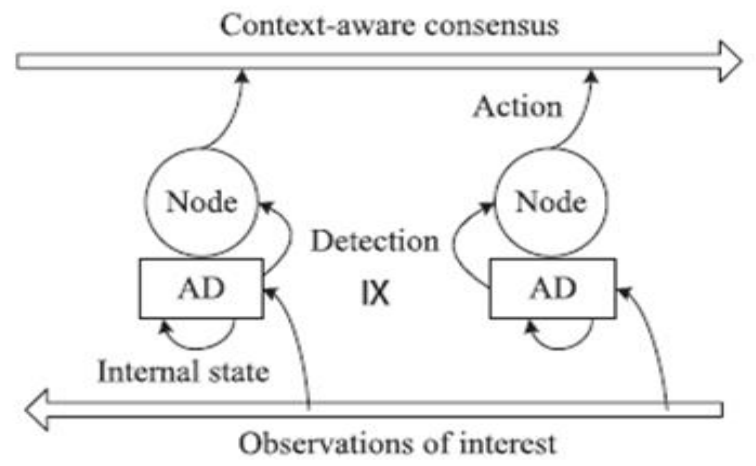

Figure 4: Individual behavior of an AD [7] 
International Journal of Network Security \& Its Applications (IJNSA) Vol. 12, No.1, January 2020

\subsection{Self-Configuration}

This aspect makes it possible to make the installation, the configuration and the integration of the complex systems within deadlines very short and without error. When an autonomous component is introduced, the autonomous networks are integrated in a homogeneously way in its environment whereas the remainder of the system adapts automatically to its presence The Selfconfiguration of the components pursues high-level policies that automatically show its capacity for adjustment. Consequently, Self-configuration is used to minimize the consumption of energy of the network and its lifespan.[1,7]

\subsection{Self-Optimization}

The networks autonomic made their Self-adjustment to maximize his performances with an aim of to make of their environment, the experimentation and the training of the choices in terms of optimization.la complexity of adjustment of the parameters can cause unforeseeable consequences on the total system.

\subsection{Self-Protection}

Because of the instability of the networks and services, the autonomous networks will have to detect the situations unforeseen and are is protected not to disturb the user.

\subsection{Self-Cure}

The size and the complexity of the networks evolve/move in a fast way from were need for developing novel methods that will be able to reach a level of service successfully. The function of the Self-cure makes it possible to repair, detect and diagnose the problems of equipment and software. In this case and to maintain the whole of functionality, the autonomous networks must be able to make decisions at a good moment. A base of knowledge on the system requirements is obligatory to make the management of maintenance and to identify the breakdowns.[1,6,7,8,9,10]

\subsection{Self - Organization}

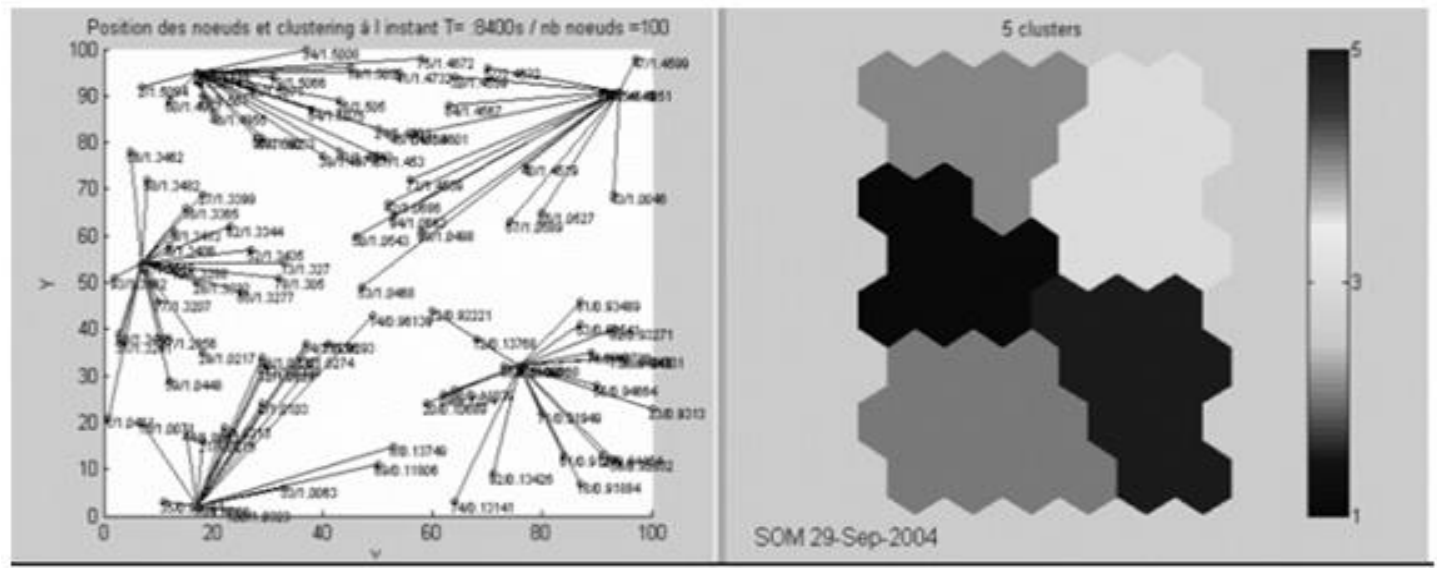

Figure 5: Visualization of the state of the network of sensors and the segmentation of the topological chart of Kohonen $\mathrm{T}=8400$ s [1] 
International Journal of Network Security \& Its Applications (IJNSA) Vol. 12, No.1, January 2020

The characteristics of the organic self-service are as follows: [1,11]

- Profit in lifespan

- Profit in quantity of message

- To observe rules of routing

- Typological visualization

- Application of an automatic treatment

\subsection{Self-management}

Self-management is based primarily on the process of training. This process begins at the end of a sequence from an output from the intentional agent. At the end of this process, the learning element gives him the context of execution. One distinguishes two types of agents, namely an agent of provisioning and an agent of monitoring [1,11].

Let us note that the element of the network makes it possible to make the Self-adaptation of its behavior. The Self-managements are articulated around 4 concepts:

-1) belief: Information on the system and the state of the environment

-2) Desires: the whole of the rules of the policies at the intermediate level

-3) Intention: plans of execution chosen by the agent of provisioning

-4) Library of plans: together preset plans

\section{ImPaCt Of Techniques OF Training For The Autonomous NETWORKS}

To learn with the networks how to adapt to various situations (software or material breakdowns) is considered as one of the major problems of the techniques of training. The choice of a method of training depends on the properties of space on assumptions. This last is structured by either a computer programming language or the application of the concept of the vicinity. In the case of the absence of assumptions, one uses the methods of closer neighbors (CBR).[1]

\subsection{Goals of the Techniques of Training for the Autonomous Networks}

- Acquisition of a base of knowledge (to conceive a plan of knowledge, which builds and maintains the models high level)

- Reflection on the success and the failure of the existing strategies

- To learn from the new strategies

- Improvement of the performances

- Improvement of knowledge

\subsection{Standards of the techniques of training for autonomous networks}

- Training supervised: As well as possible to approximate the exit wished for each entry observed

- Training not supervised: From the sample of training not labeled, one seeks subjacent regularities In the form of a function, groups of dots or complex model.

- Training by reinforcement: The data of training with reinforcement is composed of a sequence of perceptions and actions. To avoid the problem of maximization of a certain hope of 
International Journal of Network Security \& Its Applications (IJNSA) Vol. 12, No.1, January 2020

profit, it would be necessary that the autonomous networks learn the automatic reflexes and the methods of planning.

\subsection{Dynamic adaptation of the models in response times for the management of QoS applied to the autonomous networks}

In the compromise systems, the objectives of quality of service QOS are often specified by the objective service of level which stipulates a response time to reach for a percentile of the transactions. The control of the admission is of the new session's user is based on a model of response time. The dynamic approach of adaptation in response times adapts the model to the changes of the characteristics according to the workload and the system requirements so that the system can function automatically, without human intervention [12].

\section{Conclusion and Prospects}

The autonomic networks are considered as a solution for the concept of management autonomous the policies make it possible to guide the autonomous elements in terms of objectives to be reached, or service to be returned, load with this one to adapt to the dynamic modification of the rules of policy. To avoid the intervention of the human administrators, the autonomous networks have an automatic characteristic of reflection through well-defined techniques of training but the problems of safety of network (confidence of information, defense, tackles protocols of routing, the dynamic confidence of self-management...) remain stakes [12]. The management of future internet requires the adaptation of autonomous networks to intelligent strategies that provide the technical support for the construction of temporary and highly dynamic networks to meet the human needs for the future ubiquitous and flexible network [13-17]. The key components are policy-based management and intelligent systems as well as the organization of individuals who are the key actors to make Internet management work. It depends on better computation and advanced technology such as the SDN technology, which will be technological pilots for autonomous systems.

\section{REFERENCES}

[1] F. Krief, "networks autonomic," presented in conference SANDSTONE, Bordeaux, France, May. 912,2006

[2] H.Derbel, N.Agoulmine, M.Salaün. (2008, November). ANEMA: Autonomic network management structures to support coil-configuration and coil-optimization in IP networks. Networks computer. [Online]. 53 (2009), pp. 418-430.

[3] P.Ray, N.Parameswaran, L.Lewis. (18, March). Distributed autonomic management: Year Approach and experiment towards managing service-centric networks. Network and Computer Applications. [Online]. 33 (2010), pp. 653-660.

[4] J.Ermon. "Trust Estimate in autonomic networks: has a message-passing approach," presented in the First IFAC Workshop one Estimate and Control of Networked Systems, Venis, Italy, September. 2426, 2009

[5] S.Latré, J.Famaey, J.Strassner, F.Turck. (5, February) Automated context collaborative dissemination for autonomic networks through semantic subscription filter generation. Network and Computer Applications. [Online]. 36 (2013), pp. 1405-1417. 
International Journal of Network Security \& Its Applications (IJNSA) Vol. 12, No.1, January 2020

[6] D.Miorandi, L.Yamamoto, F.Pellegrini. (10, September). With a survey of evolutionary and embryogenic approaches to autonomic networking. Networks computer. [Online]. 54 (2010), pp. 944959. [12] J.Entrialgoa, D.García, J.García, M.García, P.Valledor, M.Obaidat. (5, January). Dynamic adaptation off response-time models for QoS management in autonomic systems. Systems and Software. [Online]. 84 (2011), pp. 810-820.

[7] Z.Zhang, F.N.Abdesselam, P.H. Ho, Y.Kadobayashi. (5, June). Cost-sensitive Toward coiloptimizing anomaly detection and response in autonomic networks5.com puter\&security. [Online]. 30 (2011), pp. 525-537.

[8] L.F Selfvalho, S.BarbonJr, L.S Mendes, M.L ProençaJr.Unsupervised learning clustering and coilorganized agents applied to help network management. Expert Systems With Applications. [Online]. 54 (2016), pp. 29-47.

[9] N.Tcholtchev, R.chaparadza "One coil-healing based one collaborating end-systems, access, edge and core network components," 5th International ICST Conference one Networks Access, Berlin, Germany, November. 3-5, 2010

[10] R. Sterritt. (2004, October). Autonomic networks: engineering the coil-healing property. Engineering Applications of Artificial Intelligence. [Online]. 17 (2004), pp. 727-739.

[11] J. Neuman, J. Strassner. (2008). Coil-organization and coil-management in communications ace applied to autonomic networks Computer Communications. Computer Communications. [Online]. 31 (2008), pp. 2935-2936.

[12] J.Baras. Security and Trust for Wireless Autonomic Networks Systems and Methods Control. European Journal of Control. [Online]. 13 (2007), pp. 105-133.

[13] M. Agarwal, A. Roy, N. Saxena, Next-generation 5G wireless networks: a comprehensive survey, IEEE Commun. Surv. Tutor. 18 (3) (2016) 1617-1655.

[14] K. Begum, S. Dixit, Industrial WSN using IoL: a survey, in Proceedings of the International Conference on Electrical, Electronics, and Optimization Techniques (ICEEOT), 2016, pp. 499-504.

[15] C. Yu, X. Li, W. Lin, X. Zhang, X. Gou, Feasibility Study for the LTE-Advanced System, in Proceedings of the Fourth International Conference on Advanced Information Technology and Sensor Application (AITS), 2015, pp. 80-83.

[16] F. Zhang, M. Wang, The development status and key technologies of 5G mobile communication, China New Telecommun. 6 (2016) 83-84.

[17] Z. Zhang, L. Luo, L. Wang, D2D Multicast retransmission algorithm in mobile cloud-based on SINR constraint, China Commun. 13 (8) (2016) 41-52.

\section{AUTHOR}

Houneida Sakly: Doctor and engineer in computer science. Currently, Researcher at the National School of Computer Science (ENSI) - COSMOS Laboratory and within the international medical Carthage Center in Tunisia.

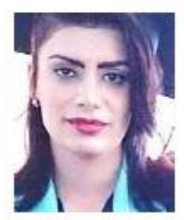

